\title{
CLOSING COMMENTS: OBSERVATIONS
}

\author{
P. M. MCCulloch \\ Physics Department, University of Tasmania
}

During the course of this colloquium many papers have been presented on observational aspects of pulsar astronomy. In the following discussion I have not attempted to be comprehensive but have selected a number of areas of interest to me.

The basic pulsar properties appear to be consistent over the full range of pulsar periods from $1 \mathrm{~ms}$ to $4 \mathrm{~s}$, implying that the emission mechanism is the same for all pulsars. There was a general consensus among the observers that the radio emission occurs low down in the pulsar's magnetosphere above the magnetic polar region.

While the basic emission process is the same, every pulsar is an individual with its own signature, a meta-stable integrated pulse profile, which differs from pulsar to pulsar. The profile should be a function of the magnetic field geometry and the line of sight. However pulsars with apparently similar geometries have different signatures suggesting that other factors influence the shape of the emission profile. There may be some way in which features on the neutron-star surface influence the emission region giving rise to individual pulse profiles. Can features exist on the surface- do neutron stars have hair? Theoreticians now believe that the work function at the neutron-star surface is insufficient to maintain a vacuum gap at the surface so that any spark formation region must be higher up, making it more difficult to explain how surface features may lead to a stable profile.

The discussion of the evolution of the magnetic field of neutron stars suggested that there was no strong evidence for significant field decay other than that due to accretion, and no evidence for evolution of inclination angle. Evidence that the field does not decay is provided by $\gamma$-ray burst sources which are thought to be old neutron stars with strong magnetic fields. If the magnetic field of a neutron star does not decay, then the problem remains: How do pulsars die, do they just stop radiating when their rotation period exceeds a few seconds?

A number of papers discussed multifrequency integrated profile observations covering a wide frequency range. Some of the observations show evidence for different dispersion measures in different frequency bands, which is interpreted in terms of non-dipolar field components or aberration and retardation effects. The available evidence is conflicting and open to different interpretations. There is a need for further careful observations possibly using microstructure, to make simultaneous measurements of the dispersion measure at a number of frequencies.

If the evidence for non-dipolar terms is supported and the magnetic field is distorted at large distances, our estimates of the size of the polar cap may need to be revised. The dipole theory may not be adequate to estimate the size of the emission region, and we may not be able to use a simple radius-to-frequency mapping. We need to understand this if we are to use a geometrical model to explain beam widths.

A number of arguments based on pulse-width statistics and position-angle data suggested that on average the emission beam is circular or slightly compressed in the axial direction. These appear to conflict with visibility and pulsar birth-rate arguments which require a fan beam.

Two of the major analytical papers were on the topic of pulsar classification, one by Rankin and the other by Lyne and Manchester. Do these classification schemes tell us something about the pulsars or are they just a variant of stamp collecting? Both schemes are based on the shape of the integrated pulse profile, but use different criteria to categorize the pulsars. Rankin argues that there are two distinct types of emission, one associated with the filled core of pulsar emission and the other with hollow cones. Young pulsars are predominantly single core-component objects, while older pulsars tend to have additional conal components, suggesting an evolutionary track. The argument is based on the relation between the pulse width $\rho$ of the various components and the pulsar period $P$.

By rigorously applying a set of criteria, Rankin is able to separate the core and conal components and finds different well defined relations for the core and the inner and outer conal components. The relations are:

$$
\begin{array}{lc}
\rho=2.45 P^{-1 / 2} & \text { core } \\
\rho=4.3 P^{-1 / 2} & \text { inner cone } \\
\rho=6.0 P^{-1 / 2} & \text { outer cone }
\end{array}
$$

Rankin interprets these as implying that the height of the emission region is independent of the pulse period but dependent on the type of component, the core emission occurring low down near the surface, while the conal emission occurs at heights betweer 
100 and $200 \mathrm{~km}$. Following on work done with Radhakrishnan, she suggests that there are two types of circular polarization. The first is seen from pulsars in which there is a change in the sense of circular polarization near the center of the profile. The sense of rotation of the position angle through the pulse is correlated with the change in the sense of the circular component, when the position angle rotates clockwise, the sense of circular changes from left to right through the profile, and vice versa. The second symmetric type of circular polarization is seen from pulsars in which the circular polarization is predominantly of one sense. Radhakrishnan and Rankin interpret the first type in terms of a geometrical effect due to an axially compressed emission beam.

Lyne argues that there are not two distinct types of emission, but just a gradation of properties. He finds no evidence for a physical difference between core and conal components. Lyne uses the maximum in the rate of change of position angle with longitucie to define the center of the pulse, and then uses symmetry arguments to estimate an overall pulse width, assuming that we do not see all of the emission cone. Lyne then finds that the pulse width is related to the pulsar period by $\rho \propto P^{-1 / 3}$ and argues that all of the emission originates at a height of about $100 \mathrm{~km}$. He also argues that there is no clear cut ring structure in the emission region, just a patchy beam. Lyne finds no evidence for two types of circular polarization, and presents 10 examples where the relation between the position angle swing and the change in sense of circular polarization is opposite that found by Radhakrishnan and Rankin.

Rankin and Lyne presented two similar but different classification schemes leading to different conclusions regarding the pulsar emission region. If the core and conal components are emitted at different heights, aberration and retardation effects should move the apparent position of the core to later longitudes with respect to the position of the center of the cone, and could even move it outside the cone. I examined about 20 triple profiles and found no examples with the central component closer to the leading cornponent but many examples where the central component is closer to the trailing component. These effects should also shift the position of the point of maximum rate of change of position angle with longitude away from center of the profile towards later longitudes. By using this maximum to define the center of the profile, Lyne seems to be assuming that all emission originates from the same height. It is not surprising therefore that Lyne and Manchester come to this conclusion.

Do we have a structured emission region consisting of a central core surrounded by a number of cones or do we have a patchy beam? The observations suggest that the number of components is either $1,2,3$ or 5 which is consistent with a ring structure. There are no pulse profiles with 4 welldefined components. Why do we only get 1, 2, 3 or 5 components if we have a patchy beam, and if the beam is patchy, why is it stable? Observations of pulsars with symmetrical three-component profiles show that the fluctuation spectra of the core and conal components are different. This suggests a key way in which these components may be identified in pulsars with asymmetric profiles.

Radhakrishnan and Rankin report one pulsar with strong circular polarization that does not fit their scheme, which may mean that the emission beam for this object is extended in latitude. The majority of observations in which the circular polarization is unambiguously defined confirm their conclusions. Many of the integrated-profile polarization observations show weak circular polarization which appears to change sense almost at random. Weak spurious circular polarization can arise in many ways and is difficult to recognize and remove unless the observer can make repeated observations at a number of different feed orientations. These observations should be confirmed with a different observing system. 